Relato de experiência - 217 -

\title{
IMPLANTAÇÃO DO SISTEMA ACOLHIMENTO COM CLASSIFICAÇÃO E AVALIAÇÃO DE RISCO E USO DO FLUXOGRAMA ANALISADOR
}

\author{
José Aparecido Bellucci Júnior', Laura Misue Matsuda²
}

${ }^{1}$ Mestrando em Enfermagem do Programa de Pós-Graduação em Enfermagem da Universidade Estadual de Maringá (UEM).
Professor do Setor de Enfermagem da Universidade Estadual do Norte do Paraná. Paraná, Brasil. E-mail: bellucci@ffalm.br
${ }^{2}$ Doutora em Enfermagem. Professora do Departamento de Enfermagem da UEM. Paraná, Brasil. E-mail: lmisue@terra.com.br

RESUMO: O presente estudo teve como objetivo relatar o processo de implantação do sistema Acolhimento com Classificação e Avaliação de Risco e o uso do Fluxograma Analisador, no Serviço Hospitalar de Emergência da Santa Casa de Misericórdia de Ourinhos, Estado de São Paulo. A implantação do sistema foi subdividida nas etapas: sensibilização dos profissionais, readequação de recursos, execução do planejamento e avaliação. À organização do fluxo de pacientes, foram utilizados Fluxogramas Funcionais que, depois de alguns ajustes, resultaram em um Fluxograma Analisador. O Fluxograma Analisador resultante proporcionou a visualização gráfica das etapas do atendimento e direcionou todos os portadores de agravos não emergenciais à consulta de enfermagem. Conclui-se que o Fluxograma Analisador foi uma ferramenta essencial ao processo de implantação do Acolhimento com Classificação e Avaliação de Risco porque, ao definir as etapas do fluxo para o atendimento, o serviço se tornou mais organizado, humano e seguro.

DESCRITORES: Enfermagem. Acolhimento. Serviço hospitalar de emergência. Qualidade da assistência à saúde. Gerência.

\section{DEPLOYMENT OF THE SYSTEM USER EMBRACEMENT WITH CLASSIFICATION AND RISK ASSESMENT AND THE USE FLOWCHAT ANALYZER}

\begin{abstract}
This study had as objective to describe the process of implementing the system User Embracement with Classification and Risk Assesment and the use of Flowchat Analyzer in Emergency Hospital Service of Santa Casa de Misericórdia de Ourinhos, state of São Paulo. The deployment of the system was subdivided into stages: professional awareness, readjustment of resources, implementation of planning and evaluation. For the organization of patient flow, Funcional Flowcharts were used which, after some adjustments, resulted in a Flowchat Analyzer. The resulting Flowchat Analyser provided a graphic display of the stages of care and directed all non-emergeny patients with injuries to the nursing consultation. It is concluded that the Flowchart Analyser was as essential tool in the process of implementation of the User Embracement with Classification and Risk Assesment because, in defining the steps of the flow to the service, the service became more organized, humane and safe.
\end{abstract}

DESCRIPTORS: Nursing. User embracement. Emergency hospital service. Quality of helth care. Management.

\section{IMPLANTAÇÃO DEL SISTEMA ACOGIMIENTO CON CLASIFICACIÓN Y EVALUACIÓN DE RIESGOS Y USO DEL FLUJOGRAMA ANALIZADOR}

RESUMEN: Este estúdio tuvo como objetivo describir el proceso de implementación del sistema Acogimiento con Clasificación y Evaluación de Riesgos y uso del Flujograma Analizador y su uso en el Servicio de Urgencias del Hospital Santa Casa de Misericordia de Ourinhos, estado de São Paulo. El despliegue del sistema se divide en etapas: la sensibilización de los profesionales, el reajuste de los recursos, la ejecución de planificación y evaluación. Para la organización del flujo de pacientes, se utilizaron Flujogramas Funcionales que, con algunos ajustes, se tradujo en un Flujograma Analizador. El Flujograma Analizador resultante proporciona una representación gráfica de las etapas de lo atención dirigida a todos los pacientes que no ha lesiones de emergencia en la consulta de enfermería. Se concluye que el Flujograma Analizador es una herramienta esencial en el proceso de aplicación del Acogimiento con Clasificación y Evaluación de Riesgos, ya que, en la definición de los pasos del flujo con el servicio, el servicio se convirtió en más organizado, humano y seguro.

DESCRIPTORES: Enfermería. Acogimiento. Servicio de urgencia en hospital. Calidad de la atención de salud. Gerencia. 


\section{INTRODUÇÃO}

No mundo globalizado, em virtude do impacto que as tendências políticas e econômicas geram na produção do conhecimento em saúde, na educação e nas condições sociais da população, torna-se cada vez mais complexo pensar na promoção da qualidade nos serviços de saúde. ${ }^{1}$

No contexto hospitalar, em especial nos Serviços Hospitalares de Emergência (SHEs), a elevada demanda de pacientes que procuram por atendimento afeta diretamente a qualidade dos serviços prestados porque as abordagens dos profissionais se tornam focadas segundo a ordem de chegada do cliente e não de acordo com o seu grau de necessidade. ${ }^{2}$ Fatos como esse, revelam a necessidade de aprofundar questões que emergem a respeito do acesso pelos usuários a esses serviços. ${ }^{3}$

No Brasil, não diferente do contexto mundial, a crescente procura por atendimento em SHEs demanda altas tecnologias e cuidados médicos e de enfermagem cada vez mais complexos e onerosos. Demandas dessa natureza consistem em desafios a serem transpostos pelos gestores e trabalhadores que primam pela qualidade da assistência pois devem adequar, cotidianamente, a estrutura e a forma de atendimento de cada serviço.

Ainda com relação ao contexto do atendimento em SHEs no Brasil, uma situação que provoca a alta demanda assistencial, além das violências e acidentes, é a procura por atendimento, de agravos que poderiam ser resolvidos na rede básica de saúde. ${ }^{4}$ Para minimizar esse problema, a literatura aponta medidas como alocação de maiores investimentos para estruturação do atendimento na rede básica, ${ }^{4-5}$ implantação de Unidades de Pronto Atendimento ${ }^{6} \mathrm{e}$ esclarecimento da população e dos profissionais que atuam na saúde sobre as reais funções de um SHE. ${ }^{7}$

No Estado de São Paulo, diferentemente de outros estados brasileiros, o atendimento de alta complexidade tem garantido a resolutividade de muitos casos por meio da rede estadual hospitalar, ${ }^{8}$ a qual consiste em um interligado de hospitais, dentro de um estado específico, que fornecem ações e serviços de prevenção e restabelecimento da saúde visando promover a integralidade na assistência ao usuário. ${ }^{9}$

Apesar de hospitais do estado de São Paulo se despontarem no atendimento a alta complexidade, quando se fala em atendimento em SHE, as dificuldades geradas pela elevada procura são semelhantes às encontradas nos outros estados brasileiros.
Frente à baixa resolutividade e à precariedade nos atendimentos efetuados pelos serviços de saúde do Brasil, o Ministério da Saúde (MS) formulou, em 2003, a Política Nacional de Humanização da Atenção e Gestão do SUS (HumanizaSUS), tendo como um dos eixos principais o Acolhimento nas ações de saúde, o qual, principalmente em relação ao atendimento em SHE, é associado ao sistema de Classificação e Avaliação de Risco. ${ }^{10-11}$

No âmbito da saúde, entende-se acolhimento como uma ação técnico-assistencial que supõe antecipadamente uma mudança na relação entre o usuário e o profissional, colocando o primeiro como participante ativo no processo de produção de saúde. ${ }^{10}$ Em SHEs, o acolhimento deve abranger os problemas de recepção de demanda fazendo junção às diretrizes de clínica ampliada, co-gestão, ambiência e valorização do trabalho em saúde, que fazem parte do HumanizaSUS, para a mudança nos processos de trabalho e gestão de serviços. ${ }^{11}$ Outra consideração importante sobre o acolhimento é que, em razão da afetividade que essa tecnologia proporciona, consiste em uma ferramenta potente para humanizar o cuidado. ${ }^{12}$

Diante dessas considerações, nota-se que é preciso discutir a melhor forma de estabelecer um fluxo de atendimento resolutivo nos SHEs, de modo que se mantenha o foco nas necessidades do usuário e, ao mesmo tempo, contemple a realidade administrativa de cada instituição.

No modelo tradicional de funcionamento dos SHEs, o cliente, muitas vezes, não é atendido de forma resolutiva, humanizada e acolhedora, em razão da alta demanda de serviços, da falta de profissionais qualificados e de organização no ambiente de trabalho. Nesse sentido, os prejuízos aos usuários podem ser inestimáveis, pois "muitos serviços de atendimento às urgências convivem com grandes filas onde as pessoas disputam o atendimento sem critério algum, a não ser a hora da chegada. A não-distinção de riscos ou graus de sofrimento faz com que alguns casos se agravem na fila, ocorrendo, às vezes, até a morte de pessoas pelo não-atendimento no tempo adequado". ${ }^{11: 22}$

Frente à necessidade de se organizar a fila de espera nos SHEs, utilizando outra ordem de atendimento que não seja a ordem de chegada, o HumanizaSUS adotou o sistema Classificação e Avaliação de Risco que, somado à diretriz do acolhimento, tem como objetivos principais garantir o atendimento imediato do usuário com grau de risco elevado; fornecer informações ao paciente e ao familiar sobre o tempo provável de 
espera. Além disso, o sistema Acolhimento com Classificação e Avaliação de Risco (ACCR) objetiva, também, minimizar a desfragmentação do processo assistencial ao proporcionar pactuções, entre redes internas e externas de atendimento a partir da construção de fluxos, de acordo com o grau de risco de cada usuário. ${ }^{10-11}$

O primeiro país a desenvolver o sistema ACCR foi a Austrália, na década de 1990. Nesse país se utilizam 5 níveis de classificação de pacientes que, do ponto de vista de espera para o atendimento, o tempo poderia variar de zero a 120 minutos. ${ }^{13-14}$

No Brasil, o sistema ACCR é realizado por enfermeiros, os quais, por meio da consulta de enfermagem, classificam os agravos com base em, no mínimo, quatro níveis ilustrados por cores ${ }^{10}$, conforme pode ser visualizado, em síntese, no quadro 1 .

\section{Quadro 1 - Níveis de classificação de risco em usuários de Pronto Socorro}

\begin{tabular}{|c|c|c|}
\hline COR & CRITÉRIO & PRIORIDADE \\
\hline VERMELHO & Emergência: necessidade de atendimento imediato & 0 \\
\hline AMARELO & Urgência: atendimento o mais rápido possível & 1 \\
\hline VERDE & Prioridade não urgente & 2 \\
\hline AZUL & Baixa complexidade: atendimento de acordo com a ordem de chegada & 3 \\
\hline
\end{tabular}

Fonte. ${ }^{9}$

Ainda com relação ao contexto da utilização do ACCR nos SHEs do Brasil, um dos hospitais pioneiros a adotar essa forma de atendimento foi o Hospital Municipal Dr. Mário Gatti em Campinas-SP, que, no ano de 2000, implantou o sistema após ser convidado pelo MS para fazer parte de um projeto piloto que visava implantar um programa de humanização em alguns hospitais brasileiros.

A partir dos bons resultados obtidos com a experiência do ACCR em Campinas-SP, outros hospitais brasileiros adotaram esse sistema. Hoje, pelos resultados satisfatórios que o ACCR tem obtido em vários hospitais, ${ }^{15-16}$ é provável que, na atualidade, esse sistema seja uma das principais propostas de reorganização de fluxo no atendimento em SHE.

Considera-se que, em razão da complexidade do trabalho desenvolvido em SHE, a prática do ACCR se constitui em grande desafio a ser transposto, uma vez que as ações para humanizar o atendimento neste serviço não acontecem somente com o "dar refúgio" ao cliente, sem que se priorize as suas necessidades de atendimento.

Para o monitoramento do processo de trabalho em SHE existe uma ferramenta denominada Fluxograma Analisador (FA) que apresenta, em detalhes, os fluxos dos pacientes e identifica os gargalos gerados no atendimento. O FA consiste em uma explanação gráfica da organização de um conjunto de processos de trabalho que se interligam entre si numa determinada cadeia de produção. ${ }^{17}$
Devido à complexidade das relações que acontecem nos processos de trabalho em um SHE, o MS enuncia o FA como uma importante ferramenta teórica para reflexão sobre a atuação da equipe na operacionalização do trabalho e na construção da relação de acolhimento com o usuário. ${ }^{2-11}$ Há, também, quem considere o FA como um instrumento potente que proporciona autoanálise e autogestão ao identificar as fragilidades e os interesses envolvidos na distribuição e na organização das instâncias de poder, relativas ao processo de trabalho. ${ }^{18}$

Como modelo de FA, sugere-se que este seja construído a partir de análises de casos quanto à entrada de pacientes, etapas percorridas, saída e resultados alcançados, de forma que cada fase do fluxo no processo de trabalho seja analisado. ${ }^{10}$ Ainda como sugestão para análise e construção do FA, recomenda-se que sejam contemplados os fatores importantes no processo como: número de atendimentos, horários de pico, fluxos de funcionamento interno, tipo de demanda, capacitação técnica dos profissionais, dentre outros. ${ }^{11}$

A utilização do FA na área da saúde tem sido relatado, com maior freqüência, nos estudos voltados às ações de prevenção em atenção primária. Apesar de também ser utilizado em alguns SHE, não foram encontrados estudos sobre a sua utilização no processo de implantação do ACCR.

Mediante a escassez de publicações referentes ao gerenciamento de fluxo em SHE utilizando o FA, associado ao fato de que, mesmo empirica- 
mente, se observa que a implantação do ACCR em um SHE do interior paulista tem produzido resultados positivos, é que se propõs a realização deste estudo.

O objetivo desse estudo foi relatar o processo de implantação do Sistema Acolhimento com Classificação e Avaliação de Risco, com uso do Fluxograma Analisador, no SHE da Santa Casa de Misericórdia de Ourinhos-SP.

O presente relato foi autorizado formalmente pela administração do hospital, foco do estudo, e a "intenção" da sua realização foi analisado e aprovado pelo Comitê Permanente de Ética em Pesquisas com Seres Humanos - COPEP, da Universidade Estadual de Maringá PR, sob o Parecer n. ${ }^{\circ} 169-10$. Vale salientar que esse trabalho recebeu apoio financeiro da Fundação Araucária: apoio ao desenvolvimento científico e tecnológico do Paraná.

\section{LOCAL DO ESTUDO}

A instituição, a qual este relato se refere, é a Santa Casa de Misericórdia de Ourinhos (SCMO), situada no interior do estado de São Paulo, e o Serviço em questão, é o Pronto Socorro, cujo atendimento às urgências e emergências acontecem em duas unidades distintas: O Pronto Socorro de Atendimento Geral (PSAG), para pacientes/clientes do Sistema Único de Saúde (SUS) e o Pronto Atendimento à Clientela Conveniada e Particular. Apesar de haver duas unidades de atendimento às urgências e emergências, devido à melhor estrutura física e de equipamentos, todas as emergências são direcionadas à primeira unidade.

O PSAG funciona desde a fundação da instituição, em 1943, possui 12 salas para atendimento, 25 leitos efetivos, é referência para 27 cidades da região, tem porta aberta durante as 24 horas do dia e atende à demanda espontânea que atinge, em média, 9.500 pacientes/mês.*

Com relação ao atendimento no PSAG, no período que precedeu o ACCR, traduzia-se em um aglomerado de pessoas, com diferentes graus de necessidades, que buscavam atendimento médico a qualquer custo, em sua grande maioria por demanda espontânea, a qual, em virtude da não priorização dos agravos para atendimento, ocasionava conflitos constantes que envolviam os próprios usuários, os profissionais, a mídia e as autoridades.

No interior do PSAG, devido aos numerosos problemas gerados pela superlotação no atendimento que, além de ocupar grande parte do tempo dos enfermeiros na resolução de conflitos, afetavam o processo de trabalho, o stress era constante entre a equipe médica, de enfermagem e usuários. As grandes filas para atendimento e a falta de informação ao usuário proporcionavam tumultos no ambiente de trabalho que refletiam diretamente na qualidade de vida dos trabalhadores os quais, frequentemente, se queixavam de descontentamentos com o serviço. Além disso, outras consequências relacionadas à saúde da equipe, de difícil mensuração, afetavam diretamente a qualidade na assistência.

\section{DESENVOLVIMENTO}

\section{Implantação do Acolhimento com Classifica- ção e Avaliação de Risco e uso do Fluxograma Analisador}

Em julho de 2007, mediante as propostas do HumanizaSUS ${ }^{2}$, foram iniciadas discussões entre o Conselho de Gestores Internos da SCMO sobre a implantação do ACCR no PSAG. A partir dessas discussões, foi criado um Grupo de Trabalho (GT), o qual ficou responsável pelo planejamento e organização das ações à implantação do sistema. O GT era composto por 1 médico, 9 enfermeiros e 20 técnicos/auxiliares de enfermagem, além do diretor clínico e dois administradores da SCMO.

O GT iniciou as ações de planejamento à implantação do ACCR, tendo como ponto de partida as ações de sensibilização aos profissionais do PSAG, os quais, durante quatro meses, por meio de oficinas de humanização, em encontros quinzenais, proporcionaram debates para disseminação dos conceitos do ACCR. Após isso, durante outros quatro meses, foram realizados treinamentos para capacitação dos funcionários do PSAG, os quais aconteceram simultaneamente, com as readequações e, também, com a construção de parte da estrutura física, a fim de proporcionar uma melhor ambiência no local.

O termo "ambiência", ainda pouco utilizado no contexto da saúde brasileira, é conceituado como o tratamento dado ao espaço físico para que este proporcione atenção acolhedora. ${ }^{10}$ Em outras

\footnotetext{
* Manual de Normas e Rotinas do Pronto Atendimento da Santa Casa de Misericórdia de Ourinhos-SP. 2009.
} 
palavras, a ambiência significa readequação da área física para que se promova o bem-estar do paciente e de seus familiares, descaracterizando o clima tenso do "ambiente hospitalar".

Mesmo com duas visitas de consultores do MS no início e no meio do processo de planejamento, e com as reuniões incansáveis do GT para desenvolver as ações de implantação, devido à inexperiência dos seus componentes, as perspectivas de resultados eram incertas, pois havia baixa adesão dos profissionais médicos e frustração dos usuários que, após várias tentativas anteriores de reorganização de fluxo, pareciam não acreditar mais em mudanças e na melhoria da vazão do atendimento.

Após oito meses de discussões e de planejamento, em março de 2008, implantou-se o ACCR no PSAG. Nos primeiros seis meses, o ACCR funcionou entre as $10 \mathrm{~h}$ e $22 \mathrm{~h}$. Depois, o horário de atendimento passou a ser das $07 \mathrm{~h}$ às $19 \mathrm{~h}$, mas em razão da necessidade de se readequar a escala de serviço dos trabalhadores da enfermagem e, também, para atender às solicitações da clientela, um novo horário de funcionamento foi acrescentado (das 19h à 01h). Atualmente, o horário de funcionamento é das $07 \mathrm{~h}$ à $01 \mathrm{~h}$.

Para que o ACCR se tornasse seguro e funcional, modificações na estrutura física e nos recursos humanos foram realizadas. Em relação à planta física, frente à necessidade de adequação à ambiência, as modificações culminaram na criação de cinco novas salas, adequadamente decoradas, localizadas na parte externa do PSAG, destinadas à aferição de SSVV, à consulta de enfermagem, e à espera pediátrica, para pacientes classificados como cor "Verde" e para os classificados como cor "Azul". Aos pacientes classificados como cor "Amarela", foi reservado um espaço na parte interna, próximo ao Posto de Enfermagem, para que pudessem ser visualizados e atendidos de imediato caso seus agravos se tornassem emergência.

Em relação à adequação na estrutura dos recursos humanos, a princípio, foram contratados dois enfermeiros e dois técnicos de enfermagem para atuar no atendimento interno do PSAG, deslocando-se o mesmo número de profissionais, com maior tempo de serviço, para atuar no ACCR. Essa conduta foi tomada porque, de acordo com o MS, todos os profissionais podem atuar no ACCR, no entanto, quando se trata de "porta de entrada", deve-se contar com profissionais preparados para a abordagem de primeiro contato ao usuário. ${ }^{11}$ Entendendo que "profissionais preparados", nor- malmente são aqueles que possuem considerável tempo de atuação em emergências e/ou os que receberam o devido treinamento para atuação no sistema, decidiu-se pela manutenção dos que atuavam a mais tempo no PSAG para compor o quadro de funcionários para o ACCR.

Além da contratação de novos profissionais de enfermagem para atuar no ACCR, foram realizadas oficinas internas e visitas a hospitais que já haviam implantado o sistema. Um dos hospitais visitados, que contribuiu sobremaneira na definição do planejamento e da logística do atendimento do PSAG da SCMO, foi o Hospital Dr. Mario Gatti de Campinas-SP, que realiza o ACCR há mais de 10 anos.

Quanto à consulta de enfermagem para a Classificação e Avaliação de Risco, mediante a possibilidade de se produzir novos gargalos no atendimento, o MS preconiza que não é necessária a realização desse procedimento com todos os pacientes que procuram pelo serviço. ${ }^{10}$

Mesmo ciente de que a referida consulta poderia aumentar o tempo de espera no atendimento, associada ao fato de que alguns profissionais da equipe de saúde a rejeitava por entender que ela - a consulta de enfermagem - é um meio que promove em demasia a autonomia do enfermeiro, a administração local, considerando o padrão de humanização e de qualidade almejado para o atendimento no PSAG, optou por estruturar o serviço de forma que todos os pacientes com agravos não emergenciais, antes do atendimento médico, passassem pela consulta de enfermagem, durante todo o período de funcionamento do ACCR.

Em maio de 2009, após 14 meses do início da sua implantação foi realizada uma pesquisa de opinião, com 125 usuários que foram atendidos no PSAG. O objetivo desse inquérito foi levantar informações sobre o atendimento nesse serviço, antes e depois da implantação do ACCR. Apesar de os dados ainda estarem em fase de tratamento e de análise, já se observa que a população reconhece que ocorreram melhorias no âmbito da humanização pela equipe de saúde, no tempo de espera e na resolutividade do atendimento.

Quanto aos profissionais que atuam no PSAG, embora de forma empírica, percebe-se que o ACCR teve boa aceitação pelos mesmos. A solicitação para ampliar o horário de atendimento e os comentários favoráveis sobre a organização no ambiente e no atendimento são alguns aspectos que merecem destaque. 
O uso do Fluxograma Analisador como ferramenta de organização do ACCR

O processo de implantação do ACCR na SCMO ocorreu num período de 24 meses subdividido em quatro etapas: sensibilização dos profissionais, readequação de recursos físicos, materiais e humanos, execução do planejamento e avaliação.

Em virtude da necessidade de reconfigurar o trabalho médico que se voltava à centralização das ações, até o ponto de ditar a forma de trabalho dos profissionais que atuavam no serviço, a readequação do fluxo de atendimento mostrou-se como uma das principais dificuldades a serem superadas nos primeiros meses. Mesmo com a baixa adesão dos médicos, o GT continuou o processo de implantação do ACCR.
Para a organização a execução das etapas da implantação do ACCR, inicialmente, foram utilizados Fluxogramas Funcionais (FFs) que são desenhos que retratam a relação entre diferentes ações de trabalho e se tornam útil no momento em que se percebe que o tempo gasto para atender o usuário é um problema.

Os FFs tiveram por finalidade monitorar as ações e as propostas articuladas às mudanças desejadas no processo de atendimento, tendo em vista que o objetivo final era chegar a um Fluxograma Analisador (FA) que proporcionasse, com maior precisão, a visualização gráfica de todas as etapas do atendimento.

No início da implantação do ACCR, o FA era assim constituído:

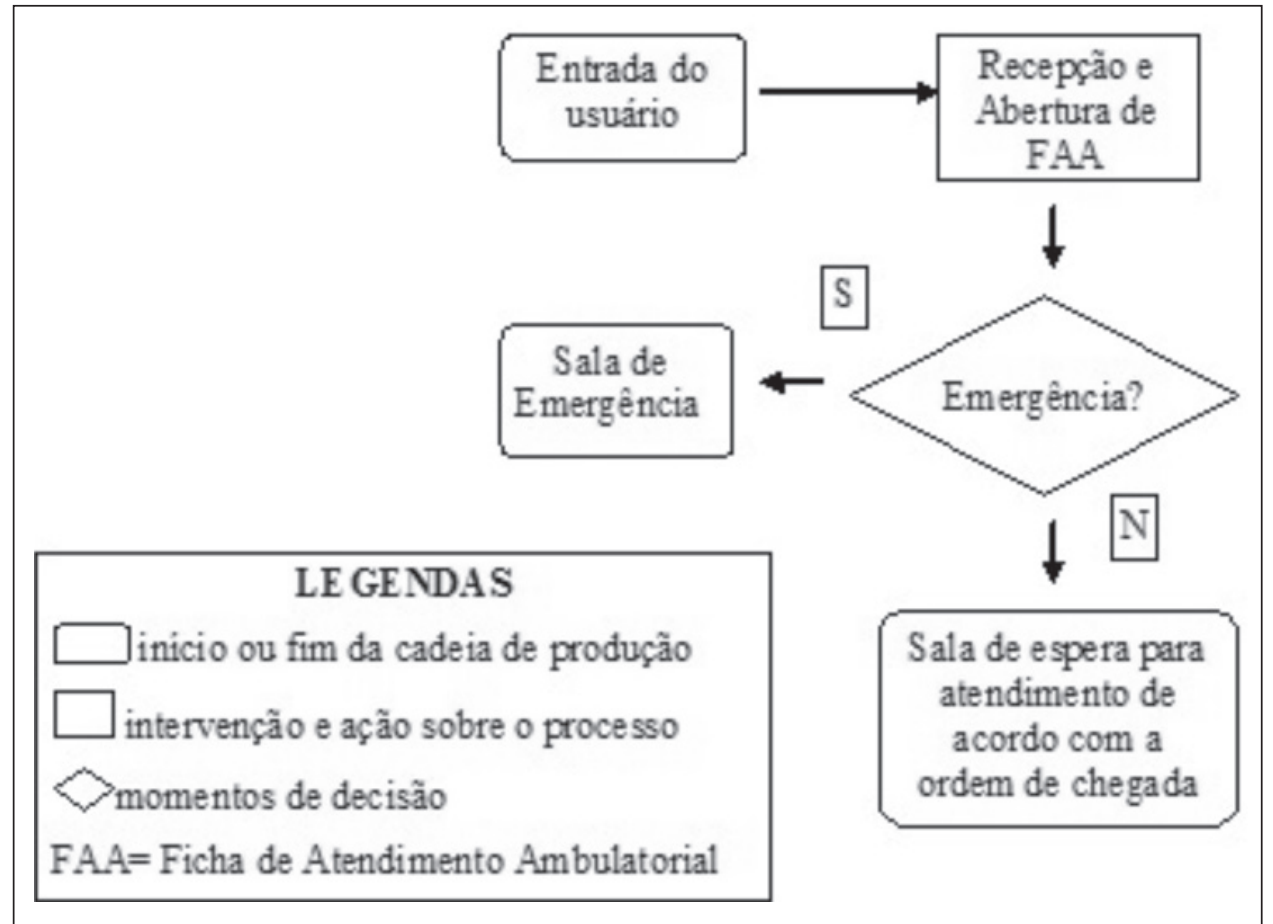

Figura 1 - Fluxograma Analisador antes da implantação do ACCR. Ourinhos-SP, 2010

Em diversos momentos, os FFs foram modificados de acordo com as necessidades/problemas apresentados.

O primeiro problema foi observado por meio da aglomeração de pessoas em um único local, ou seja, na sala de espera, em que os clientes/ pacientes aguardavam atendimento, de acordo com a ordem de chegada, sem que lhes fossem dada a devida importância quanto ao grau de necessidade.

O segundo problema se referia à ocorrência da "pseudo" Classificação de Risco, no serviço de recepção, realizado por um profissional sem competência para tal, que muitas vezes discernia uma emergência baseando-se no estado aparente e/ou naquele caso em que o paciente e/ou familiar "falava mais alto".

Apesar de resistências às mudanças serem manifestadas por vários trabalhadores, partiu-se para a discussão e construção de um novo fluxograma, o Analisador (FA) à recepção do usuário (Figura 2), de modo que todas as etapas do $\mathrm{ACCR}^{10}$ fossem contempladas. Nesse sentido, de maneira objetiva o fluxograma resultante representa o fluxo de atendimento ao usuário na Santa Casa de Misericórdia de Ourinhos. 


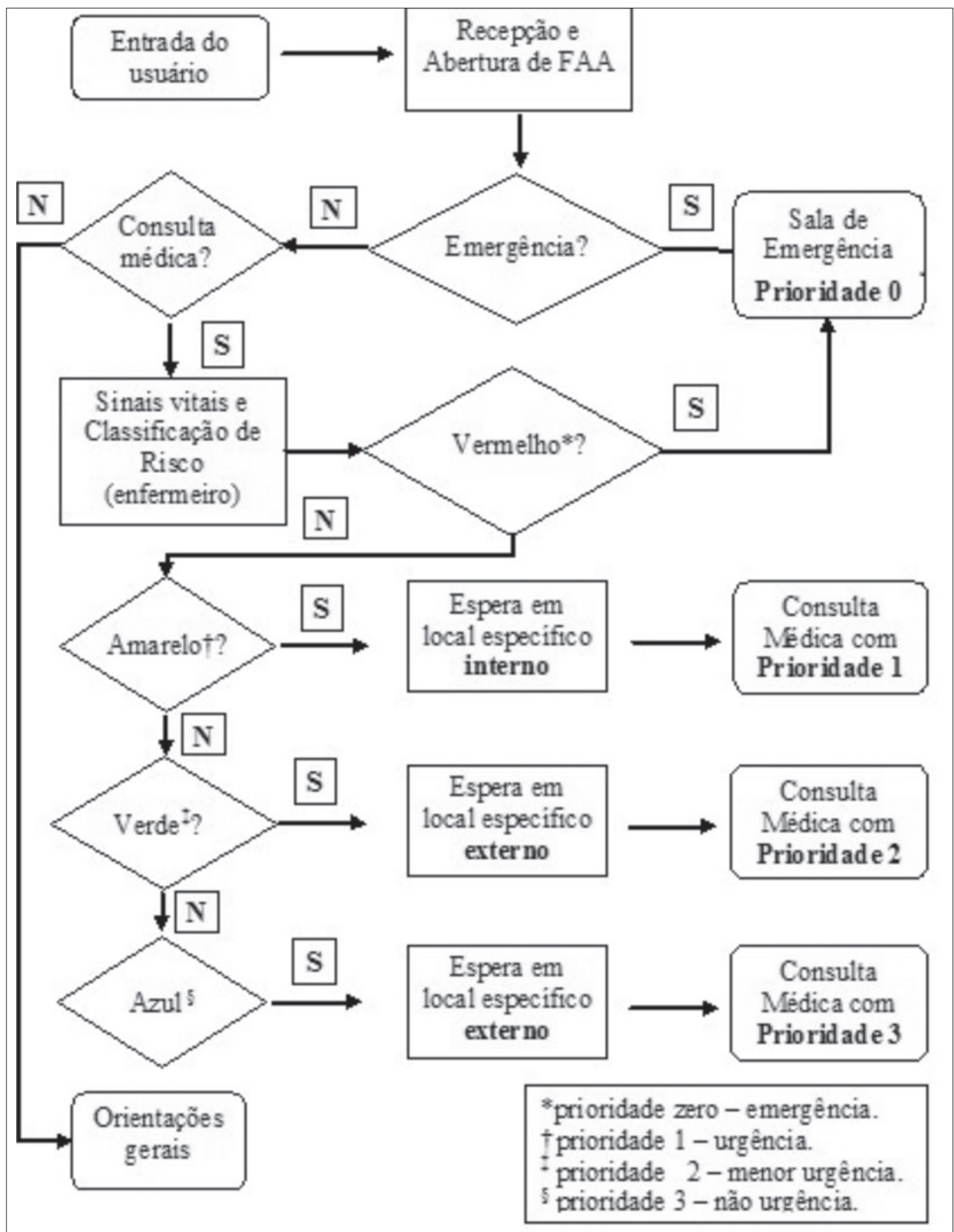

Figura 2 - Fluxograma Analisador atual do ACCR da SCMO. Ourinhos-SP, 2010

Na figura 2 é possível observar que a consulta de enfermagem é realizada a todos os portadores de agravos não emergenciais que procuram o serviço. Após a verificação dos sinais vitais pelo técnico em enfermagem e a Classificação de Risco pelo enfermeiro, que acontece depois da referida consulta, e cujas cores são identificadas por meio de adesivos colocados nas fichas de atendimento, dependendo das condições, o paciente é direcionado a uma sala para atendimento específico e informado sobre o seu grau de urgência e prioridade no atendimento.

No período inicial da implantação do ACCR, em razão do desconhecimento de como o sistema funcionava, a população reagiu com certa indignação e, por se sentir desprestigiada, reclamava ao Serviço de Ouvidoria do hospital. No entanto, após seis meses da implantação, já era possível observar que o ACCR passara a ser mais bem aceito porque as Fichas de Avaliações dos usu- 
ários e as conversas informais entre os diversos profissionais que atuavam no setor revelavam esse dado. Além disso, outros indícios comprovam que houve melhora no atendimento ao usuário nesse serviço: diminuição dos casos de agressividade dos pacientes enquanto em espera por consulta médica, redução do número de reclamações na ouvidoria da SCMO, com relação ao atendimento de porta, redução da sobrecarga de serviço dos recepcionistas, diminuição do tempo de espera pelo atendimento médico, entre outros.

\section{CONSIDERAÇÕES FINAIS}

Mais do que acolhimento com Classificação e Avaliação de Risco, a implantação desse sistema e sua monitoração pelo FA proporcionou gestão com qualidade e para a qualidade do PSAG da SCMO. As manifestações de que houve organização do Serviço, associado à melhoria na humanização do atendimento aos clientes e dos profissionais, parecem comprovar o resultado do trabalho desenvolvido pelo GT e pelos demais trabalhadores da instituição.

Quanto à adesão dos profissionais ao programa, no início, a classe médica pouco aderiu à proposta, porém, com o passar do tempo, mediante a gradativa compreensão da realidade prática do ACCR, principalmente após o primeiro semestre, a aceitação ganhou força. Atualmente, de forma surpreendente, declarações como "não sei como trabalhávamos naquela época" e "hoje não trabalharíamos sem o ACCR" são facilmente manifestadas nos encontros de profissionais, como é o caso das reuniões do Conselho Gestor Interno realizadas semanalmente na instituição.

Ao falar em qualidade do atendimento, em especial em SHE, é necessário que, além das intenções, aconteçam muitas ações de todos os níveis hierárquicos e de seus trabalhadores para que o resultado não seja nada menos que o "descongestionamento" e a resolução dos casos, de acordo com a necessidade de cada agravo, em seu devido tempo e local. Nessa perspectiva, à implantação e consolidação do ACCR na SCMO, há que se destacar alguns fatores que seguramente impulsionaram todo o processo: desejo da alta direção em humanizar e qualificar o atendimento; investimentos para melhorar a estrutura física (ambiência) e de recursos humanos; busca pela melhoria contínua dos processos de atendimento e envolvimento gradativo dos trabalhadores, em especial, da equipe de enfermagem.
Dentre os fatores que dificultaram - e ainda dificultam - o desenvolvimento do ACCR, constam: elevado número de pacientes com agravos que poderiam ser atendidos na rede básica de saúde, mas que continuam procurando pelo SHE, a necessidade de contratação/remoção de mais profissionais de enfermagem para atuação nos horários de picos e criação de novas salas para atendimento.

Considera-se que o sistema de Acolhimento com Classificação e Avaliação de Risco implantado no PSAG da SCMO ainda necessita de muitos ajustes, mas, mediante a definição clara das etapas que compõem o fluxo de atendimento, refletido no FA desse serviço, associado à priorização da atenção aos que apresentam maior agravo, o serviço se tornou mais organizado, humano e seguro.

\section{REFERÊNCIAS}

1. Duarte MSM, Silvino ZR. Acreditação hospitalar $x$ qualidade dos serviços de saúde. R Pesq: Cuid Fundam Online. 2010 Out-Dez; 2(Supl):182-5.

2. Ministério da Saúde (BR). Política Nacional de Humanização da Atenção e Gestão em Saúde HumanizaSUS. Brasília (DF): MS; 2004.

3. Souza ACC, Moreira TMM, Silva MRF, Almeida PC. Acesso ao serviço de emergência pelos usuários com crise hipertensiva em um hospital de Fortaleza, CE, Brasil. Rev Bras Enferm. 2009 Jul-Ago; 62(4):535-9.

4. Deslandes SF, Minayo MCS, Lima MLC. Atendimento de emergência às vítimas de acidentes e violências no Brasil. Rev Panam Salud Publica 2008; 24(6):430-40.

5. Mendes ACG, Araújo JR JLCA, Furtado BMAS, Duarte PO, Santiago RF, Costa TR. Avaliação da satisfação dos usuários com a qualidade do atendimento nas grandes emergências do Recife, Pernambuco, Brasil. Rev Bras Saúde Matern. Infant. 2009 Abr-Jun; 9(2):157-65.

6. Bittencour RJ, Hortale VA. Intervenções para solucionar a superlotação nos serviços de emergência hospitalar: uma revisão sistemática. Cad Saúde Pública. 2009 Jul; 25(7):1439-5.

7. Oliveira MLF, Scocchi MJ. Determinantes da utilização do serviço de urgência/emergência em Maringá (PR). Cienc Cuid Saúde. 2002 Jan-Jun; 1(1):123-8.

8. Souza RR, Mendes JDV, Portas SLC, Barros S, Vallim S. Plano estadual de saúde 2008 - 2011. São Paulo: Secretaria da Saúde, 2008.

9. Ministério da Saúde (BR), Conselho Nacional das Secretarias Municipais de Saúde. O SUS de A a Z: garantindo saúde nos municípios. $3^{\mathrm{a}}$ ed. Brasília (DF): MS; 2009. 
10. Ministério da Saúde(BR), Secretaria Executiva Núcleo Técnico da Política Nacional de Humanização. HumanizaSUS: acolhimento com avaliação e classificação de risco: um paradigma ético-estético no fazer em saúde. Brasília (DF): MS; 2004.

11. Ministério da Saúde (BR), Secretaria de Atenção à Saúde, Acolhimento e classificação de risco nos serviços de urgência. Política nacional de humanização da atenção e gestão do SUS. Brasília (DF): MS; 2009.

12. Rossi FR, Lima MADL. Acolhimento: tecnologia leve nos processos gerenciais do enfermeiro. Rev Bras Enferm. 2005 Mai-Jun; 58(3):305-10.

13. Nishio EA, Urrutia M, Araújo JE. Análise pela lógica difusa da condição de risco e sinais vitais para atendimento em Unidade de Emergência. In: Anais do $11^{\circ}$ Congresso Brasileiro de Informática em Saúde; 2008 Nov 29-Dez 03; Campos do Jordão, Brasil. (SP): SBIS; 2008. DVD.

14. Murray M, Bullard M, Michael MD, Grafstein E. Revisions to the Canadian Emergency Department
Triage and Acuity Scale Implementation Guidelines. CJEM. 2004 Nov; 6(6):421-7.

15. Ministério da Saúde [página na Internet]. Brasília (DF): MS; 2009 [atualizado 2010 fevereiro 11; acesso 2010 Jun 28]. Disponível em: www.saude.gov.br

16. Grupo Brasileiro de Acolhimento com Classificação de Risco. Hospital João XXIII divulga resultado após implantação de Protocolo; 2009 [acesso 2009 Fev 25]. Disponível em: http://www.gbacr.com.br/index. php?option $=$ com_content\&task $=\&$ id $=99 \& I t e m i d=1$

17. Malta DC, Ferreira LM, Reis AT, Merhy EE. Acolhimento: uma reconfiguração do processo de trabalho em saúde usuário-centrada. In: Campo CR, Malta DC, Reis AT, Santos AF, Merhy EE, organizadores. Sistema Único de Saúde em Belo Horizonte: reescrevendo o público. São Paulo (SP): Xamã, 1998. p.121-42.

18. Barboza TAV, Fracolli LA. A utilização do "fluxograma analisador" para a organização da assistência à saúde no Programa Saúde da Família. Cad Saúde Pública. 2005 Jul-Ago; 21(4):1036-44. 